TRANSACTIONS OF THE

AMERICAN MATHEMATICAL SOCIETY

Volume 28I. Number 1. January 1984

\title{
A TWO WEIGHT WEAK TYPE INEQUALITY FOR FRACTIONAL INTEGRALS
}

\author{
BY
}

ERIC SAWYER

ABSTRACT. For $1<p \leqslant q<\infty, 0<\alpha<n$ and $w(x), v(x)$ nonnegative weight functions on $R^{n}$ we show that the weak type inequality

$$
\int_{\left\{T_{\alpha} f>\lambda\right\}} w(x) d x \leqslant A \lambda^{-q}\left(\int|f(x)|^{p} v(x) d x\right)^{q / p}
$$

holds for all $f \geqslant 0$ if and only if

$$
\int_{Q}\left[T_{\alpha}\left(\chi_{Q} w\right)(x)\right]^{p^{\prime}} v(x)^{1-p^{\prime}} d x \leqslant B\left(\int_{Q} w\right)^{p^{\prime} / q^{\prime}}<\infty
$$

for all cubes $Q$ in $R^{n}$. Here $T_{\alpha}$ denotes the fractional integral of order $\alpha, T_{\alpha} f(x)=$ $\int|x-y|^{\alpha-n} f(y) d y$. More generally we can replace $T_{\alpha}$ by any suitable convolution operator with radial kernel decreasing in $|x|$.

1. Introduction. Weighted norm inequalities for fractional integrals have been treated by several authors. For example, B. Muckenhoupt and R. L. Wheeden have shown [8] that the one weight strong type inequality

$$
\left(\int_{R^{n}}\left|T_{\alpha} f(x) w(x)\right|^{q} d x\right)^{1 / q} \leqslant C\left(\int_{R^{n}}|f(x) w(x)|^{p} d x\right)^{1 / p} \quad \text { for all } f \geqslant 0
$$

where $1 / q=1 / p-\alpha / n$ holds if and only if $w(x)^{q}$ satisfies the $A_{r}$ condition with $r=1+q / p^{\prime}$. Here $T_{\alpha} f(x)=\int_{R^{n}}|x-y|^{\alpha-n} f(y) d y$ is the fractional integral or Riesz potential of order $\alpha$ (see [10] for the basic properties of $T_{\alpha}$ ) and the $A_{r}$ condition on a function $v(x)$ is

(A $) \quad\left(\int_{Q} v(x) d x\right)^{1 / r}\left(\int_{Q} v(x)^{-r^{\prime} / r} d x\right)^{1 / r^{\prime}} \leqslant C \int_{Q} d x$ for all cubes $Q$

where the second factor on the left side is interpreted as $\left\|\chi_{Q} v^{-1}\right\|_{\infty}$ in the case $r=1$. In a different direction, B. Dahlberg [3] has used a capacitary strong type inequality to show that a positive measure $\omega$ satisfies the "trace" inequality

$$
\int_{R^{n}}\left|T_{\alpha} f(x)\right|^{p} d \omega(x) \leqslant C \int_{R^{n}}|f(x)|^{p} d x \quad \text { for all } f \geqslant 0
$$

Received by the editors March 22, 1983.

1980 Mathematics Subject Classification. Primary 42B25.

${ }^{1}$ Research supported in part by NSERC grant \# A5149. 
if and only if

$$
\omega(E) \leqslant A \operatorname{Cap}(E)=A \inf \left\{\int|f(x)|^{p} d x ; T_{\alpha} f \geqslant 1 \text { on } E\right\}
$$

for all compact subsets $E$ of $R^{n}$. See also D. Adams [1] and V. Maz'ya [7] for the case $\alpha$ integral. More recently, R. Kerman and the author [6] (see also [9]) have shown that (2) is equivalent to the simpler condition

$$
\int_{Q}\left|T_{\alpha}\left(\chi_{Q} \omega\right)(x)\right|^{p^{\prime}} d x \leqslant C \int_{Q} d \omega<\infty \quad \text { for all cubes } Q \text {. }
$$

However, the characterization of the general two weight strong type inequality for fractional integrals remains open. In this note we address the simpler two weight weak type inequality and give a characterization of it in terms of a condition analogous to (4). As in [5 and 6] we will treat operators more general than fractional integrals, namely convolution operators of the form $T f=K * f$ where $K(x)$ is a positive radial function decreasing in $|x|$. K. Hansson has recently obtained a capacitary strong type inequality for such operators [5] and hence the equivalence of (2) and (3) for $T$ in place of $T_{\alpha}$ (the corresponding equivalence of (2) and (4) is in [6]). If $\mu$ is a positive measure on $R^{n}$ we use the notation $|E|_{\mu}=\int_{E} d \mu$ and $T(f \mu)(x)=K *(f \mu)(x)=\int K(x-y) f(y) d \mu(y)$.

Theorem. Suppose $1<p \leqslant q<\infty, \omega$ and $\mu$ are positive borel measures on $R^{n}$ and $T f=K * f$ where $K(x)$ is a positive lower semicontinuous radial function decreasing in $|x|$. If $n \geqslant 2$ suppose, in addition, that $K(x)$ satisfies $\left(A_{1}\right)$. Then the weak type inequality

$$
|\{T(f \mu)>\lambda\}|_{\omega} \leqslant A \lambda^{-q}\left(\int|f|^{p} d \mu\right)^{q / p} \text { for all } f \geqslant 0, \lambda>0,
$$

holds if and only if

$$
\int_{Q}\left|T\left(\chi_{Q} \omega\right)\right|^{p^{\prime}} d \mu \leqslant B|Q|_{\omega}^{p^{\prime} / q^{\prime}}<\infty \quad \text { for all cubes } Q .
$$

Furthermore if $A$ and $B$ are the least such constants, then the ratio $A^{1 / q} / B^{1 / p^{\prime}}$ is bounded between two positive constants independent of $\omega$ and $\mu$.

REMARKS. I. The theorem is also valid for $p=1$ if (6) is replaced by $\left\|T\left(\chi_{Q} \omega\right)\right\|_{L^{\infty}(\mu)} \leqslant C|Q|_{\omega}^{1 / q^{\prime}}<\infty$ for all cubes $Q$.

II. The result stated in the abstract follows from the Theorem with $K(x)=|x|^{\alpha-n}$, $d \omega(x)=w(x) d x, d \mu(x)=v(x)^{1-p^{\prime}} d x$ and $f$ replaced by $f v^{p^{\prime}-1}$. Note that $|x|^{\alpha-n}$ satisfies $\left(A_{1}\right)$ for $0<\alpha<n$.

III. If $\mu$ is an $A_{\infty}$ weight then condition (6) is sufficient for the strong type analogue of (5) (see [6]) but, in general, condition (6) is not sufficient. See D. Adams [1, Remark 2(iii)] for a counterexample in the case $p=q$ (note that ( $\left.\mathrm{a}^{\prime}\right), \mathrm{p} .134$ in [1] is equivalent to (6) with $d \omega=d x, T=T_{m}$ and $p^{\prime}, q^{\prime}$ replaced by $q, p$, respectively) and $\S 3$ below for the case $p \leqslant q$. 
2. Proof of the Theorem. Assume (5) holds. Provided $\mu$ is nontrivial $\left(0<|E|_{\mu}<\infty\right.$ for some set $E$ ) the positivity of $K$ together with (5) easily shows that $|Q|_{\omega}<\infty$ for all cubes $Q$. The remaining inequality in (6) is an easy consequence of duality (of Lorentz spaces). In fact,

$$
\begin{aligned}
& \left(\int\left|T\left(\chi_{Q} \omega\right)\right|^{p^{\prime}} d \mu\right)^{1 / p^{\prime}}=\sup _{\|f\|_{L^{p_{(}(\mu)}} \leqslant 1} \int T\left(\chi_{Q} \omega\right) f d \mu=\sup _{\|f\|_{L^{p^{(}(\mu)}} \leqslant 1} \int_{Q} T(f \mu) d \omega \\
& =\sup _{\|f\|_{L, \rho_{(\mu)}} \leqslant 1} \int_{0}^{\infty}|Q \cap\{T(f \mu)>\lambda\}|_{\omega} d \lambda \\
& \leqslant \int_{0}^{\infty} \min \left\{A \lambda^{-q},|Q|_{\omega}\right\} d \lambda \text { by }(5) \\
& =q^{\prime} A^{1 / q}|Q|_{\omega}^{1 / q^{\prime}}
\end{aligned}
$$

and so (6) holds with $B \leqslant\left(q^{\prime}\right)^{p^{\prime}} A^{p^{\prime} / q}$.

Conversely, suppose (6) holds and, without loss of generality, that $f$ is nonnegative with compact support and satisfies $\int|f|^{p} d \mu<\infty$. The main idea of the proof is to establish a "good $\lambda$ inequality" (in much the same manner as is done in R. Coifman [2]) for $T f$ relative to the maximal operator

$$
M f(x)=\sup _{x \in Q} \frac{1}{|Q|_{\omega}} \int_{Q} T\left(\chi_{Q} f\right) d \omega .
$$

We begin with the case $n=1$. Fix $0<\beta<1$ and $\lambda>0$. Since $T f \mu$ is lower semicontinuous we can write $\{T f \mu>\lambda\}=\dot{\cup}_{k} I_{k}$ where the intervals $I_{k}=\left(a_{k}, b_{k}\right)$ are disjoint. Moreover, (6) implies that $\left|I_{k}\right|_{\omega}<\infty$ for all $k$ (if $I_{k}$ is infinite, then $\lim _{x \rightarrow \infty} K(x)>0$ and it is easy to see that (6) implies $\left.\int_{-\infty}^{\infty} d \omega<\infty\right)$. We now discard those $I_{k}$ with $\left|I_{k}\right|_{\omega}=0$ and denote by $F$ the set of indices $k$ such that

$$
\frac{1}{\left|I_{k}\right|_{\omega}} \int_{I_{k}} T\left(\chi_{I_{k}} f \mu\right) d \omega>\beta \lambda
$$

and by $G$ the set of $k$ for which (7) fails. For $k$ in $F$ we have

$$
\begin{aligned}
\lambda^{q}\left|I_{k}\right|_{\omega} & <\beta^{-q}\left|I_{k}\right|_{\omega}^{1-q}\left(\int_{I_{k}} T\left(\chi_{I_{k}} f \mu\right) d \omega\right)^{q} \\
& =\beta^{-q}\left|I_{k}\right|_{\omega}^{1-q}\left(\int_{I_{k}} T\left(\chi_{I_{k}} \omega\right) f d \mu\right)^{q} \\
& \leqslant \beta^{-q}\left|I_{k}\right|_{\omega}^{1-q}\left(\int_{I_{k}} T\left(\chi_{I_{k}} \omega\right)^{p^{\prime}} d \mu\right)^{q / p^{\prime}}\left(\int_{I_{k}}|f|^{p} d \mu\right)^{q / p} \\
& \leqslant \beta^{-q} B^{q / p^{\prime}}\left(\int_{I_{k}}|f|^{p} d \mu\right)^{q / p} \text { by }(6) .
\end{aligned}
$$


Now observe that if $\tilde{I}_{k}$ denotes the complement of $I_{k}$ then $T\left(\chi_{I_{k}} f \mu\right) \leqslant 2 \lambda$ on $I_{k}$ by the maximum principle. Indeed, if $x$ is in $I_{k}$ then

$$
\begin{aligned}
T\left(\chi_{\tilde{I}_{k}} f \mu\right)(x) & =\left(\int_{-\infty}^{a_{k}}+\int_{b_{k}}^{\infty}\right) K(x-y) f(y) d \mu(y) \\
& \leqslant \int_{-\infty}^{a_{k}} K\left(a_{k}-y\right) f(y) d \mu(y)+\int_{b_{k}}^{\infty} K\left(b_{k}-y\right) f(y) d \mu(y) \\
& \leqslant T f\left(a_{k}\right)+T f\left(b_{k}\right) \leqslant 2 \lambda .
\end{aligned}
$$

Thus for $k$ in $G$

$$
\begin{aligned}
\left|I_{k} \cap\{T(f \mu)>3 \lambda\}\right|_{\omega} & \leqslant\left|I_{k} \cap\left\{T\left(\chi_{I_{k}} f \mu\right)>\lambda\right\}\right|_{\omega} \\
& \leqslant \frac{1}{\lambda} \int_{I_{k}} T\left(\chi_{I_{k}} f \mu\right) d \omega \leqslant \beta\left|I_{k}\right|_{\omega}
\end{aligned}
$$

since (7) fails. Combining (8) and (9) we obtain the "good $\lambda$ inequality"

$$
\begin{aligned}
(3 \lambda)^{q}|\{T(f \mu)>3 \lambda\}|_{\omega} & =\sum_{k}(3 \lambda)^{q}\left|I_{k} \cap\{T(f \mu)>3 \lambda\}\right|_{\omega} \\
& \leqslant 3^{q} \sum_{k \in F} \lambda^{q}\left|I_{k}\right|_{\omega}+3^{q} \lambda^{q} \sum_{k \in G}\left|I_{k} \cap\{T(f \mu)>3 \lambda\}\right|_{\omega} \\
& \leqslant\left(\frac{3}{\beta}\right)^{q} B^{q / p^{\prime}} \sum_{k \in F}\left(\int_{I_{k}}|f|^{p} d \mu\right)^{q / p}+3^{q} \beta \lambda^{q} \sum_{k \in G}\left|I_{k}\right|_{\omega} \\
& \leqslant\left(\frac{3}{\beta}\right)^{q} B^{q / p^{\prime}}\left(\int|f|^{p} d \mu\right)^{q / p}+3^{q} \beta \lambda^{q}|\{T(f \mu)>\lambda\}|_{\omega}
\end{aligned}
$$

since $q / p \geqslant 1$. Choose $\beta=\frac{1}{2}\left(\frac{1}{3}\right)^{q}$ and take the supremum in (10) over $0<\lambda \leqslant t / 3$ to obtain

$$
\sup _{0<\lambda \leqslant t} \lambda^{q}|\{T(f \mu)>\lambda\}|_{\omega} \leqslant \frac{3^{q^{2}+q}}{2^{q}} B^{q / p^{\prime}}\left(\int|f|^{p} d \mu\right)^{q / p}+\frac{1}{2} \sup _{0<\lambda \leqslant t} \lambda^{q}|\{T(f \mu)>\lambda\}|_{\omega}
$$

for all $t>0$. If we can show that the left side of (11) is finite for all $t>0$, we can subtract the second term on the right side of (11) from both sides to obtain (5) with $A \leqslant 3^{q^{2}+q} B^{q / p^{\prime}} / 2^{q-1}$. To see that the left side of (11) is finite suppose that $f$ is supported in an interval $I=(a, b)$ and that $r>2 b-a$. Then from (6) we have

$$
B\left(\int_{a}^{r} d \omega\right)^{p^{\prime} / q^{\prime}} \geqslant \int_{a}^{b}\left|T\left(\chi_{(a, r)} \omega\right)(x)\right|^{p^{\prime}} d \mu(x) \geqslant \int_{I}\left|K(r-x) \int_{a}^{r} d \omega\right|^{p^{\prime}} d \mu(x)
$$


and so $\left(\int_{I} K(r-x)^{p^{\prime}} d \mu(x)\right)\left(\int_{a}^{r} d \omega\right)^{p^{\prime} / q} \leqslant B$. Thus for $r>2 b-a$ and $\lambda=T(f \mu)(r)$ we have

$$
\begin{aligned}
\lambda^{q}|\{T(f \mu)>\lambda\} \cap(a, \infty)|_{\omega} & \leqslant\left(\int_{I} K(r-x) f(x) d \mu(x)\right)^{q} \int_{a}^{r} d \omega \\
& \leqslant\left(\int_{I} f^{p} d \mu\right)^{q / p}\left(\int_{I} K(r-x)^{p^{\prime}} d \mu(x)\right)^{q / p^{\prime}} \int_{a}^{r} d \omega \\
& \leqslant\left(\int_{I} f^{p} d \mu\right)^{q / p} B^{q / p^{\prime}}<\infty .
\end{aligned}
$$

Similarly one can show that for $\lambda$ sufficiently small

$$
\lambda^{q}|\{T(f \mu)>\lambda\} \cap(-\infty, b)|_{\omega} \leqslant\left(\int_{I} f^{p} d \mu\right)^{q / p} B^{q / p^{\prime}}<\infty
$$

and this shows that the left side of (11) is finite for $t>0$ and completes the proof of the Theorem in the case $n=1$.

We now turn to the case $n \geqslant 2$ and assume that $K$ satisfies the $\left(A_{1}\right)$ condition. Recall that $f$ is nonnegative with compact support and satisfies $\int|f|^{p} d \mu<\infty$. Again fix $0<\beta<1$ and $\lambda>0$. Let $M f$ denote the maximal function of $f$, i.e.

$$
M f(x)=\sup _{x \in Q} \frac{1}{|Q|} \int_{Q}|f|
$$

and for $r>0$ denote by $r Q$ the cube concentric with $Q$ and having $r$ times the side length of $Q$. Using a variant of the Whitney covering lemma in C. Fefferman $[4, \mathrm{p}$. 16] we can write $\Omega_{\lambda}=\{M(T(f \mu))>\lambda\}=\dot{U}_{k} Q_{k}$ where the cubes $Q_{k}$ satisfy both a Whitney condition

$$
r Q_{k} \cap \tilde{\Omega}_{\lambda} \neq 0 \text { for all } k
$$

and a finite overlap condition

$$
\sum_{k} \chi_{2 Q_{k}} \leqslant D \chi_{\Omega_{\lambda}}
$$

where $r$ and $D$ are positive constants depending only on the dimension $n$.

Since $K$ satisfies $\left(A_{1}\right)$ and is decreasing as a function of $|x|$ it is easy to show that

$$
K(x) \leqslant C K(y) \text { for }|y| \leqslant 2|x| .
$$

Fix $k$ for the moment and let $f_{1}=f \chi_{2 Q_{k}}$ and $f_{2}=f-f_{1}$. For $x$ in $Q_{k}$ we have

$$
\begin{aligned}
T\left(f_{2} \mu\right)(x) & =\int_{y \notin 2 Q_{k}} K(x-y) f(y) d \mu(y) \\
& \leqslant C^{\prime} \int_{y \notin 2 Q_{k}}\left(\frac{1}{\left|Q_{k}\right|} \int_{Q_{k}} K(z-y) d z\right) f(y) d \mu(y) \quad \text { by }(14) \\
& \leqslant \frac{C^{\prime}}{\left|Q_{k}\right|} \int_{Q_{k}} T(f \mu)(z) d z \\
& \leqslant C^{\prime} r^{n} \frac{1}{\left|r Q_{k}\right|} \int_{r Q_{k}} T(f \mu)(z) d z \leqslant C^{\prime} r^{n} \lambda \quad \text { by }(12) .
\end{aligned}
$$


Thus for $\gamma>2 C^{\prime} r^{n}$ we have

$$
\{T(f \mu)>\gamma \lambda\} \cap Q_{k} \subset\left\{T\left(\chi_{2 Q_{k}} f \mu\right)>\frac{1}{2} \gamma \lambda\right\} \cap Q_{k} .
$$

Denote by $F$ the set of indices $k$ such that

$$
\frac{1}{\left|2 Q_{k}\right|_{\omega}} \int_{2 Q_{k}} T\left(\chi_{2 Q_{k}} f \mu\right) d \omega>\beta \lambda
$$

and by $G$ the set of $k$ for which (16) fails. Using (15) and arguing as in the case $n=1$ (see (8) and (9)) we obtain

$$
\left|\{T(f \mu)>\gamma \lambda\} \cap Q_{k}\right|_{\omega} \leqslant \max \left\{\beta\left|2 Q_{k}\right|_{\omega},(\beta \lambda)^{-q} B^{q / p^{\prime}}\left(\int_{2 Q_{k}} f^{p} d \mu\right)^{q / p}\right\}
$$

for all $k$ and then summing over $k$ and using (13) we get

$$
|\{T(f \mu)>\gamma \lambda\}|_{\omega} \leqslant \beta D\left|\Omega_{\lambda}\right|_{\omega}+(\beta \lambda)^{-q} B^{q / p^{\prime}}\left(D \int f^{p} d \mu\right)^{q / p}
$$

for $\gamma>2 C^{\prime} r^{n}$ and $0<\beta<1$. However $T(f \mu)$ satisfies $\left(A_{1}\right)$ with the same constant $C$ that works for $K$ and so $M(T(f \mu)) \leqslant C T(f \mu)$. Thus $\left|\Omega_{\lambda}\right|_{\omega} \leqslant|\{T(f \mu)>\lambda / C\}|_{\omega}$ and if we use this inequality on the first term on the right side of (17) we obtain an analogue of the good $\lambda$ inequality (10) and the proof can now be completed as in the case $n=1$.

3. An example. Fix $p=2 \leqslant q<\infty, n=1$ and $1 / 2 \leqslant \alpha+1 / q<1$ with $\alpha>0$. We construct a pair of weights $w, v$ on the real line satisfying the condition in the abstract $(p=2)$

$$
\int_{Q}\left[T_{\alpha}\left(\chi_{Q} w\right)\right]^{2} v^{-1} \leqslant B\left(\int_{Q} w\right)^{2 / q^{\prime}} \text { for all intervals } Q
$$

but not the corresponding strong type inequality

$$
\left(\int\left|T_{\alpha} f\right|^{q} w\right)^{1 / q} \leqslant C\left(\int|f|^{2} v\right)^{1 / 2} \text { for all } f \geqslant 0 .
$$

Let

$$
f(x)=x^{-1}|\log x|^{-q^{\prime}} \chi_{(0,1 / 2)}(x)
$$

and set $v(x)=f(x)^{-1}$ and $w(x)=x^{q-q \alpha-1}|\log x|^{q^{\prime}-1} \chi_{(0,1 / 2)}(x)$. Since $T_{\alpha} f(x) \approx$ $x^{\alpha-1}|\log x|^{1-q^{\prime}}$ we have that the left side of (19) is infinite while the right side is finite. On the other hand, using the estimates

$$
\left\|T_{\alpha}\left(\chi_{(a, r)} w\right)\right\|_{\infty} \leqslant(r-a)^{\alpha} r^{q-q \alpha-1}|\log r|^{q^{\prime}-1}
$$

and

$$
\int_{a}^{r} x^{-1}|\log x|^{-q^{\prime}} d x \underset{\sim}{\sim} \begin{cases}|\log r|^{1-q^{\prime}}, & 0 \leqslant a<r / 2 \\ |\log r|^{-q^{\prime}}\left(\frac{r-a}{r}\right), & r / 2 \leqslant a<r\end{cases}
$$


which are valid for $1 / 2 \leqslant \alpha+1 / q<1, \alpha>0$ and $0 \leqslant a<r \leqslant 1 / 2$ we obtain

$$
\int_{a}^{r}\left[T_{\alpha}\left(\chi_{(a, r)} w\right)\right]^{2} v^{-1} \leqslant(r-a)^{2 / q^{\prime}} r^{2 q+2 \alpha-2 q \alpha-2-2 / q^{\prime}|\log r|^{2-2 / q^{\prime}}} \leqslant\left(\int_{a}^{r} w\right)^{2 / q^{\prime}}
$$

for $0 \leqslant a<r \leqslant 1 / 2$ which is (18).

\section{REFERENCES}

1. D. R. Adams, On the existence of capacitary strong type estimates in $R^{n}$, Ark. Mat. 14 (1976), $125-140$.

2. R. R. Coifman, Distribution function inequalities for singular integrals, Proc. Nat. Acad. Sci. U.S.A. 69 (1972), 2838-2839.

3. B. Dahlberg, Regularity properties of Riesz potentials, Indiana Univ. Math. J. 28 (1979), 257-268.

4. C. Fefferman, Inequalities for strongly singular convolution operators, Acta Math. 124 (1970), 9-36.

5. K. Hansson, Imbedding theorems of Sobolev type in potential theory, Math. Scand. 45 (1979), 77-102.

6. R. Kerman and E. Sawyer, Weighted norm inequalities of trace-type for potential operators, preprint.

7. V. G. Maz'ya, On some integral inequalities for functions of several variables, Problems in Math. Analysis, No. 3, Leningrad Univ. (Russian)

8. B. Muckenhoupt and R. L. Wheeden, Weighted norm inequalities for fractional integrals, Trans. Amer. Math. Soc. 192 (1974), 251-275.

9. E. Sawyer, Multipliers of Besov and power weighted $L^{2}$ spaces, Indiana Univ. Math. J. (to appear).

10. E. M. Stein, Singular integrals and differentiability properties of functions, Princeton Univ. Press, Princeton, N.J., 1970.

Department of Mathematical Sciences, McMaster University, Hamilton, Ontario L8S 4K1 CANADA 